\title{
There is a movement towards the development of hula costume CAD
}

\author{
Taketo Kamasaka, Kenji Sakoma, Kodai Miyamoto, Makoto Sakamoto*, Amane Takei \\ Faculty of Engineering, University of Miyazaki, \\ Miyazaki-City, Miyazaki, Japan \\ Tsutomu Ito \\ National Institute of Technology, Ube College \\ Ube-City, Yamaguchi, Japan \\ Takao Ito \\ Graduate School of Engineering, Hiroshima University \\ Higashi-Hiroshima, Hiroshima, Japan
}

E-mail:hm16011@student.miyazaki-u.ac.jp,hm15037@student.miyazaki-u.ac.jp,hm16043@student.miyazaki-u.ac.jp,
fruits2000jp@yahoo.co.jp
*Corresponding Author

\begin{abstract}
In recent years, three-dimensional computer graphics (3DCG) technology has been applied in various fields. One of the problems in this technology is the problem of contact between clothes and other objects (such as the body). In this paper, we verified each simple animation using the three methods of calculation. As a result of the verification, the FB Euler method creates a simple but stable animation Was completed. In this paper, we were able to create simple animations.
\end{abstract}

\section{Introduction}

In recent years, 3D computer graphics (3DCG) technology has been applied in various fields such as AR / VR technology, movies, games, and virtual fitting of clothes ${ }^{1}$.

In this research, we will use a physical model to simulate cloth, which is applied to 3DCG animation.

In this research, we focus on past research and work on a simulation of a simple cloth (for example, a skirt) that considers contact with other objects (the body of a hula dancer) $)^{2,3,4}$.

\section{Simulation method}

(C) The 2021 International Conference on Artificial Life and Robotics (ICAROB2021), January 21 to 24, 2021
The Modeling methods for expressing cloth can be roughly divided into two types: geometric models and physical models.

In order to perform dynamic simulation, we will use a physical model that considers the change process this time. 


\subsection{Mass spring model}

In the mass spring model, in order to express the anisotropy of the cloth, the shape of the cloth is a spring that connects the mass points arranged in a square grid and the mass points adjacent to each other with the direction along the warp and weft threads of the cloth as the axis. (See Fig. 1.).

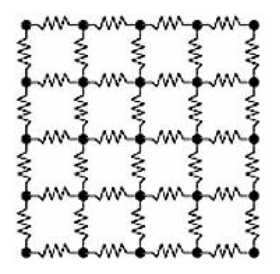

Fig. 1. Mass spring model

\subsection{Mechanical properties of cloth}

Of the six mechanical properties of cloth listed by KES, four are considered when modeling the shape of cloth: tensile properties, shear properties, bending properties, and weight properties (See Fig. 2.) ${ }^{5}$.

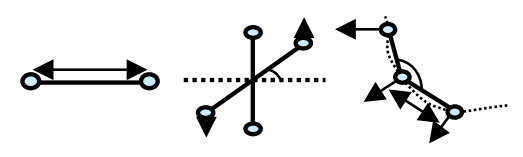

Fig. 2. Mechanical propeties of cloth

\subsection{Power on mass points}

The resultant force $\boldsymbol{F}_{\boldsymbol{i}}(\boldsymbol{t})$ of the force applied to the particle $i$ at time $t$ is as follows from the external force such as wind force $\boldsymbol{f}_{\text {wind }}$ and gravity $\boldsymbol{f}_{\text {gravity }}$ and the force generated from the internal structure of the cloth such as the damping force $\boldsymbol{f}_{\text {damper }}$ against the vibration of the spring and the force $\boldsymbol{f}_{\text {stretch }}$ by the spring. Can be expressed in

$$
\begin{gathered}
\boldsymbol{F}_{\boldsymbol{i}}(t)=\boldsymbol{f}_{\text {gravity }}+\boldsymbol{f}_{\text {dampar }}+\boldsymbol{f}_{\text {stretch }}+\boldsymbol{f}_{\text {wind }} \\
=m_{i} \boldsymbol{g}+\boldsymbol{r}_{\boldsymbol{i}}(t)+\boldsymbol{f}_{\boldsymbol{i}}(t)+\alpha \cos (\beta) \sin (\gamma)+\alpha \sin (\beta) \sin (\gamma) .
\end{gathered}
$$

\subsection{Equation of motion}

Based on the equation of motion, the acceleration $\boldsymbol{a}_{\boldsymbol{i}}(t)$ is expressed by the following equation.

$$
\boldsymbol{a}_{\boldsymbol{i}}(t)=\boldsymbol{F}_{i}(t) / m_{i} \text {. }
$$

\subsection{Numerical calculation method}

In this study, Euler method, FB Euler method, and Runge-Kutta method were used from the explicit formulas 6 .

\subsubsection{Euler method}

Assuming that the mass point moves linearly with constant acceleration between time $t$ and time $t+\Delta t$ the velocity $\boldsymbol{v}_{\boldsymbol{i}}$ and the coodinates $\boldsymbol{x}_{\boldsymbol{i}}$ can be calculated by the following equations using the force acting on the particles calculated in Section 2.2.

$$
\begin{gathered}
\boldsymbol{v}_{i}(t+\Delta t)=\boldsymbol{v}_{i}(t)+\boldsymbol{a}_{i}(t) \Delta t \\
\boldsymbol{x}_{i}(t+\Delta t)=\boldsymbol{x}_{i}(t)+\boldsymbol{a}_{i}(t) \Delta t \\
(\Delta t: \text { time step) }
\end{gathered}
$$

\subsubsection{FB Euler method}

It is a numerical calculation method that uses both the Euler method and the backward Euler method at the same time. Velocity applies the Euler method for current acceleration, and position applies the backward Euler method for future speed.

$$
\begin{gathered}
\boldsymbol{v}_{i}(t+\Delta t)=\boldsymbol{v}_{i}(t)+\boldsymbol{a}_{i}(t) \Delta t \\
\boldsymbol{x}_{i}(t+\Delta t)=\boldsymbol{x}_{i}(t)+\boldsymbol{a}_{i}(t) \Delta t .
\end{gathered}
$$

$(\Delta t$ : time step)

\subsubsection{Runge-Kutta method}

At first glance, the amount of calculation seems to be four times, but since the calculation accuracy is high, the time step width $\Delta \mathrm{t}$ can be increased, and more stable simulation is possible.

$$
\begin{gathered}
k \boldsymbol{v}_{\mathbf{1}}=\boldsymbol{a}_{\mathbf{1}} \Delta t, k \boldsymbol{x}_{\mathbf{1}}=\boldsymbol{v}(t) \Delta t \\
\boldsymbol{a}_{\mathbf{2}}=\frac{F\left(\boldsymbol{x}(t)+k \boldsymbol{x}_{\mathbf{1}} / 2\right)}{m}-c \boldsymbol{v}(t)+\boldsymbol{g} \\
k \boldsymbol{v}_{\mathbf{2}}=\boldsymbol{a}_{\mathbf{2}} \Delta t, k \boldsymbol{x}_{\mathbf{2}}=\left(\boldsymbol{v}(t)+k \boldsymbol{v}_{\mathbf{1}} / 2\right) \Delta t \\
\boldsymbol{a}_{\mathbf{3}}=\frac{F\left(\boldsymbol{x}(t)+k \boldsymbol{x}_{\mathbf{2}} / 2\right)}{m}-c \boldsymbol{v}(t)+\boldsymbol{g} \\
k \boldsymbol{v}_{\mathbf{3}}=\boldsymbol{a}_{\mathbf{3}} \Delta t, k \boldsymbol{x}_{\mathbf{3}}=\left(\boldsymbol{v}(t)+k \boldsymbol{v}_{2} / 2\right) \Delta t \\
\boldsymbol{a}_{\mathbf{4}}=\frac{F\left(\boldsymbol{x}(t)+k \boldsymbol{x}_{\mathbf{3}} / 2\right)}{m}-c \boldsymbol{v}(t)+\boldsymbol{g} \\
k \boldsymbol{v}_{\mathbf{4}}=\boldsymbol{a}_{\mathbf{4}} \Delta t, k \boldsymbol{x}_{\mathbf{4}}=\left(\boldsymbol{v}(t)+k \boldsymbol{v}_{3}\right) \Delta t \\
\boldsymbol{v}(t+\Delta t)=\boldsymbol{v}(t)+\left(\frac{1}{6} k \boldsymbol{v}_{\mathbf{1}}+\frac{1}{3} k \boldsymbol{v}_{\mathbf{2}}+\frac{1}{3} k \boldsymbol{v}_{\mathbf{3}}+\frac{1}{6} k \boldsymbol{v}_{\mathbf{4}}\right) \\
\boldsymbol{x}(t+\Delta t)=\boldsymbol{x}(t)+\left(\frac{1}{6} k \boldsymbol{x}_{\mathbf{1}}+\frac{1}{3} k \boldsymbol{x}_{\mathbf{2}}+\frac{1}{3} k \boldsymbol{x}_{\mathbf{3}}+\frac{1}{6} k \boldsymbol{x}_{\mathbf{4}}\right) .
\end{gathered}
$$

$(\Delta t$ : time step) 
The motion of mass points in the virtual world can be simulated by these equations.

\subsection{Collision with other objects}

In this study, we consider the collision between the cloth and the body. Since a large number of mass points and the body must be judged, a penalty method that takes a simple calculation is used. This time, the mass point is regarded as a point and the bone is regarded as a capsule, and collision judgment is performed.

\subsubsection{Expression of the body}

A capsule is a cylinder with hemispheres attached to both ends, defined by a line segment and radius $r$, and is the part within the distance and radius $r$ from this line segment.

Calculating the position of the capsule with the back, hips, thighs, and shin bones as the axis of the cylinder did not perform collision detection correctly(see Fig. 3.). Therefore, this time, in order to increase the number of capsules and improve the accuracy of collision detection, the back and shin bones are divided into two, and the thigh bones are divided into three.

\subsubsection{Penalty method}

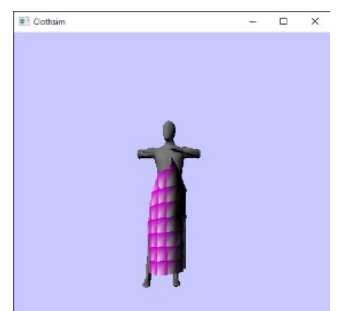

Fig. 3. Execution result by FB Euler method

The penalty method is one of the rigid body simulation methods. When objects collide with each other and a slight sunk occurs, a force (penalty force) proportional to the sunk value can be applied to obtain the behavior after the collision (See Fig.3.).

From this, it is possible to push back the lattice points colliding with the cloth to the outside of the body by the force of the spring so as not to be sunk into the body.

\subsubsection{Collision detection with capsule point}

The reason why capsules are used for collision detection is that the judgment only needs to be replaced. The collision between the capsule and the point is divided into a columnar part and a hemispherical part at both ends. Let the point $x$ for collision determination be the intersection of the perpendicular and the line segment drawn from the point $x$ to the line segment: the point $y$ is obtained. At this time, the coordinates $a$ and $b$ at both ends of the line segment are set, and since the point $y$ is on the line passing through the coordinates $a$ and $b$, it is expressed by the following equation.

$$
\boldsymbol{y}=\boldsymbol{a}+s(\boldsymbol{b}-\boldsymbol{a}) .
$$

$$
\text { (a, b, } \boldsymbol{y}: 3 \mathrm{D} \text { vector, } s: \text { Scalar) }
$$

Since the perpendicular line is orthogonal to this line, it is necessary to satisfy the following equation.

$$
\begin{gathered}
(\boldsymbol{y}-\boldsymbol{x}) \cdot(\boldsymbol{b}-\boldsymbol{a})=0 . \\
(\boldsymbol{x}: 3 \mathrm{D} \text { vector })
\end{gathered}
$$

The only unknown is $s$, and when $s$ is found, the point $y$ is found, and it is possible to determine the collision between the cylinder and the foot. At this time, if the point $y$ exists on the line segment and the distance $r^{\prime}$ between the point $x$ and the point $y$ is smaller than $r$, it can be seen that the point $y$ collides with the cylinder. Since $r-r^{\prime}$ is the distance from the surface of the cylinder to the point $x$, this value is calculated using the penalty method.

If it is found that the cylinder does not collide, then the collision between the point and the sphere is determined. The collision between a point and a sphere can be determined by examining whether the distance between the points $a$ and $b$ and the point $x$ is within $r$. Collision determination between a capsule and a sphere (radius $r^{\prime \prime}$ ) is based on the same principle, and the above $r$ is replaced with $r+r$ for calculation and determination.

\section{Simulation environment}

This program was created using the DirectX SDK, which is a standard CG library, in a program development environment using the $\mathrm{C} / \mathrm{C}++$ language. Microsoft Visual Studio Community 2019 was used to create the program this time. It ran on Intel Core i7 (Windows 10 Pro).

\section{Simulation result}


The FB Euler method is used as the numerical calculation method. The simulation results are shown in the figure below (See Fig. 4.).

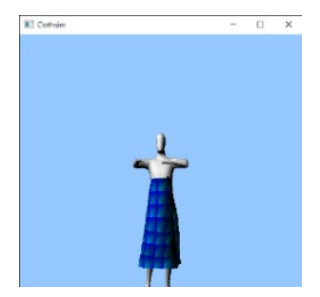

Fig. 4. Execution result by FB Euler method using the proposed method

Simulations were performed using the Euler method and the Runge-Kutta method. The Euler method resulted in the cloth moving violently and moving away from the body (See Fig. 5.). In the Runge-Kutta method, the movement of the animation became heavy, and the result was that the contact between the foot and the skirt caused the animation to dig in (See Fig. 6.).

In addition, all the calculation methods resulted in the animation moving being heavier than the execution result when the bones were not divided.

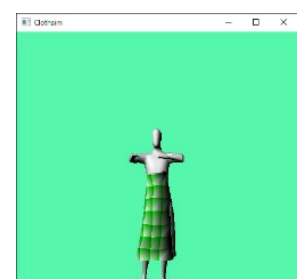

Fig. 5. Execution result by Euler method using the proposed method

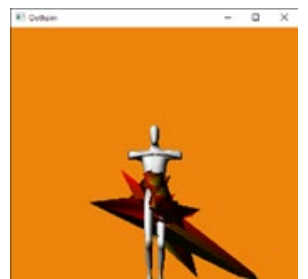

Fig. 6. Execution result by Runge-Kutta method using the proposed method

\section{Consideration}

As a result of the simulation, the collision judgment considering the contact between the skirt and the body was stable by the FB Euler method as mentioned in the execution result of Section 4, and a simple animation could be performed. However, there are some issues:

- The parameters must be changed manually.

- Examination of numerical calculation method and contact force calculation method by implicit solution method.

- Examining the method of texture mapping to the skirt.
- Examination of a method with a small amount of calculation

\section{Consideration}

The purpose of this study was to dynamically express the cloth and to try a simple animation considering the contact between the body and the skirt with reference to past studies.

This study, the completed animation is a simple animation, the number of quality points is small, and there are many parts that cannot be implemented such as texture mapping and texture expression of cloth. It is still difficult to do a realistic clothing simulation. Furthermore, we must pay attention to how to put clothes on 3D characters, how to express the difference in cloth materials, how to judge collisions with higher accuracy, and how to calculate less.

In addition, including the improvement measures mentioned above, we will work on CAD production that can simulate various movements of hula and dress design, etc., and express more realistic cloth, which will be a practical research. I want to aim for that.

\section{References}

1. Zorah Lahnar, Daniel Cremers and Tony Tung DeepWrinkles: Accurate and Realistic Clothing Modeling, 15th European Conference on Computer Vision, 2018, pp. 1-18.

2. Ayumi Taniue, Attempt to create cloth animation with OpenGL, Memoirs of the Faculty of Engineering, University of Miyazaki, 2007, pp. 299-304.

3. Seira Nagatomo, Attempt of cross simulation by mass spring model, Memoirs of the Faculty of Engineering, University of Miyazaki, 2009, pp. 327-332.

4. Minaho Sakumoto, Attempt of cloth animation considering contact with an object, Memoirs of the Faculty of Engineering, University of Miyazaki, 2011, 223-227.

5. Mari Inoue, Research on the Mechanics of Fibrous Materials, SEN'I GAKKAISHI(Textiles and industry). Vol. 64. No.8, ,2008, pp.246-251.

6. Mikihiro Satou, Research on interactive cloth simulation, Outline of the 37th Academic Lecture, Faculty of Industrial Science and Technology, Nihon University. No.20, 2004, pp.23-26. 